\title{
Severe Neuropathy and Tetraparesis Induced by Adalimumab
}

\author{
Zeljko Vucicevic $^{\mathrm{a}, \mathrm{c}}$, Vesna Degoricija ${ }^{\mathrm{a}}$, Lidija Dezmalj-Grbelja ${ }^{\mathrm{b}}$
}

\begin{abstract}
We report a case of severe axonal and demyelinating peripheral polyneuropathy with consecutive tetraparesis following the second application of adalimumab in a 55-year-old man with rheumatoid arthritis. The treatment provided $40 \mathrm{mg}$ of adalimumab s.c. every 2 weeks. Modest muscle pain and weakness followed the first dose, but the second dose resulted in progressive tetraparesis and complete immobility. Electroneurography revealed almost complete denervation of all muscles of the upper extremities, and no motor response at the lower extremities. The finding was suggestive of severe subchronic axonal and demyelinating peripheral polyneuropathy induced by adalimumab. A comprehensive medical work out excluded the possibility of other etiology. At discharge, methylprednisolone was continued for the next 3 months. Nine months of physical rehabilitation and supportive treatment resulted in a modest recovery. Anti-TNF- $\alpha$ agents may induce vasculitis neuropathy at any time during treatment which must be distinguished from the neuropathy of the underlying disease. Appropriate therapy should be initiated immediately and continued long enough.
\end{abstract}

Keywords: Anti-TNF- $\alpha$ neuropathy; Adalimumab neuropathy; Anti-TNF- $\alpha$ vasculitis

\section{Introduction}

Elevated levels of proinflammatory cytokine tumor necrosis

Manuscript accepted for publication February 12, 2013

${ }^{a}$ University Department of Medicine, Sestre Milosrdnice University Hospital Center, Zagreb, University of Zagreb, School of Medicine, Vinogradska cesta 29, HR-10000 Zagreb, Croatia

${ }^{b}$ University Department of Neurology, Sestre Milosrdnice University Hospital Center,Zagreb, University of Zagreb, School of Medicine, Vinogradska cesta 29, HR-10000 Zagreb, Croatia

${ }^{\mathrm{c} C}$ Corresponding author: Zeljko Vucicevic, University Department of Medicine, Sestre Milosrdnice University Hospital Center, University of Zagreb, School of Medicine, Vinogradska cesta 29, HR-10000 Zagreb, Croatia. Email: zeljko.vucicevic@zg.t-com.hr

doi: http://dx.doi.org/10.4021/jnr189e factor alpha (TNF- $\alpha)$ were detected in patients with various immune diseases [1]. TNF- $\alpha$ inhibition represents a significant advance in the treatment of rheumatoid arthritis, and is useful in the treatment of ankylosing spondylitis, psoriatic arthritis, Crohn's disease and ulcerative colitis [2]. Adalimumab is a human recombinant IgG1 anti-TNF monoclonal antibody that binds specifically to soluble and membranebound TNF- $\alpha$ and inhibits its interaction with the p55 and p75 cell surface TNF receptors [3]. Serious neurologic side effects suggestive of demyelination have been reported in patients receiving adalimumab or other anti-TNF agents $[4$, 5]. We describe an early and severe axonal and demyelinating peripheral polyneuropathy with consecutive tetraparesis following the second application of adalimumab in a patient with rheumatoid arthritis.

\section{Case Report}

A 55-year-old man with rheumatoid arthritis, who had unsuccessfully been treated with standard medications for 24 months, started taking adalimumab in January 2010 (40 $\mathrm{mg} / 2$ weeks). Modest, progressive pain and weakness of the limb muscles, low-back pain, and weight loss ( $4 \mathrm{~kg} / 2$ weeks) slowly followed the first dose of adalimumab.

He still received the second dose two weeks later, which subsequently resulted in tetraparesis, complete immobility, and serious deterioration of general condition. On admission, he was febrile $39.0^{\circ} \mathrm{C}$, cachectic, stuporous, and dehydrated with severe skeletal muscles hypotrophy. Laboratory data included: C-reactive protein $294 \mathrm{mg} / \mathrm{L}$, erythrocyte sedimentation rate $61 \mathrm{~mm} / 1$ st hour, white blood cell count $16.7 \times 10^{9} / \mathrm{L}$ with a "shift to left", platelet count $599 \times 10^{9} / \mathrm{L}$, international normalized ratio 1.31 (prothrombin time 56\%), fibrinogen $8.4 \mathrm{~g} / \mathrm{L}$, creatine phosphokinase $1,760 \mathrm{~J} / \mathrm{L}$, AST $89 \mathrm{U} / \mathrm{L}$, total serum protein $5.1 \mathrm{~g} / \mathrm{dL}$, albumin $1.53 \mathrm{~g} / \mathrm{dL}$, globulins $(\mathrm{g} / \mathrm{dL})$ : $\alpha_{1} 0.66, \alpha_{2} 0.88, \beta 0.84, \gamma 1.17$. The ferritin was $530 \mu \mathrm{g} / \mathrm{L}$ (normal for men $30-400 \mu \mathrm{g} / \mathrm{L}$ ).

Other rutine laboratory tests, chest radiography, ECG, abdominal ultrasonography, echocardiography, MRI scan of the brain and brain stem were within the normal range. A normal level of procalcitonin, negative bacteriological speci- 
mens and normal leukocyte count following volume replacement were indicative of nonbacterial inflammation.

Neurologic examination was suggestive of severe flaccid tetraparesis of all 4 extremities. Biochemical and bacteriological findings of cerebrospinal fluid (CSF) were normal. Electroneurography (ENG) was done on the third day of admission to reveal only a minimal voluntary activity of the left biceps and complete denervation of all other muscles of the upper extremities. At the lower extremities, no motor response was detected on any nerve explored. The ENG finding spoke in favor of severe subchronic axonal and demyelinating peripheral polyneuropathy of almost all peripheral nerves associated with consecutive severe muscular hypotrophy.

The exacerbation of rheumatoid vasculitis, associated polymyositis, neurotoxic effect of adalimumab induced vasculitis or a combination of these factors were considered on differential diagnosis. The rheumatoid factor was highly increased $1,518 \mathrm{IU} / \mathrm{mL}$ (normal $\leq 14$ ) as were circulating immune complexes $\operatorname{IgG} 1,020 \mathrm{mg} / \mathrm{L}$ (normal $\leq 130)$ and $\operatorname{IgM}$ $1,415 \mathrm{mg} / \mathrm{L}$ (normal $\leq 100$ ). For suspicion of adalimumab induced vasculitis, methylprednisolone was started at a dose of $1 \mathrm{mg} / \mathrm{kg} / \mathrm{b}$.w. Only 24 hours later, the patient became afebrile, and inflammatory parameters soon returned to normal.

Polymyositis was excluded by ENG, serologic test, and muscle biopsy. Anti-dsDNA, anti-nuclear antibodies, anti-neutrophil cytoplasmic antibody, anti-myeloperoxidase antibodies, autoantibodies to proteinase 3 , anti-cyclic citrullinated peptide antibody, anti Jo1, lupus anticoagulant, anticardiolipin antibodies, tests for cytomegalovirus, toxoplasmosis, HIV infection, antistreptolysin O, antistaphylolysin, thyroid hormones, and tumor markers were within the normal range. At discharge, methylprednisolone was continued for the next 3 months. Continuous physical therapy and symptomatic treatment were recommended.

Nine months later, neurologic examination indicated modest recovery of proximal segments and residual paresis of the fists and feet associated with significant muscular hypotrophy. ENG still showed severe distal polyneuropathy accompanied by the signs of complete denervation and satisfactory recovery of proximal segments comparing with previous finding.

\section{Discussion}

An important step in the pathogenesis of inflammatory demyelination is the invasion of the peripheral nervous system by activated, circulating, autoreactive T cells [6]. TNF- $\alpha$ is released by these $\mathrm{T}$ cells or macrophages and acts at several stages in the development of inflammatory demyelination [7]. It exerts cytotoxic damage to vascular endothelium and a breakdown of the blood-nerve barrier that results in increased vascular permeability, which facilitates access to the nerve microenvironment of circulating factors such as immunoglobulins, cytokines, and complement $[7,8]$. TNF- $\alpha$ may cause selective cytotoxic damage to Schwann cells and myelin sheats [9], and may also represent a major noxious molecule by which macrophages damage peripheral nerve in Guillain-Barre syndrome [7].

Literature data associate anti-TNF- $\alpha$ therapy with a variety of central nervous system demyelinating disease, and peripheral neuropathy syndromes [10]. Cases of optic neuritis [11], mononeuritis multiplex [12], and multiple sclerosis [5] following adalimumab therapy were described. In different reports, the timing of the onset of neuropathy relative to TNF- $\alpha$ inhibitor infusion varied considerably from several hours after the first infusion to 2 years of continuous treatment with adalimumab $[5,10,13]$.

The treatment and recovery depends on the type of neuropathy, severity of damage, and therapeutic approach, and may last for weeks or months [10]. Complete anti-TNF therapy cessation is not always necessary and may be limited to patients with ongoing disabling neuropathies and controlled underlying affections [14]. Patients with chronic inflammatory demyelinating polyradiculoneuropathy, multifocal motor neuropathy, axonal sensory and sensorimotor neuropathies may improve spontaneously over a period of several months [10]. Methylprednisolone alone seems to be insufficient in Guillain-Barre syndrome and Miller-Fisher syndrome, or in multifocal motor neuropathy and vasculitic mononeuropathy multiplex [10]. Concurrent treatment with cyclophosphamide or IVIG is recommended.

Severe axonal peripheral polyneuropathy was predominant in our patient, which usually requires only supportive therapy. Nine months after discharge, only a modest recovery was reached. Some case reports and uncontrolled studies suggest that anti-TNF- $\alpha$ therapy is effective in the treatment of various types of systemic vasculitis but at the same time, vasculitis is the most common autoimmune disease triggered by the TNF-targeted therapies [15]. TNF- $\alpha$ inhibitors are optimal for patients with vasculitis refractory to steroids and immunosuppressants, especially if they were not previously treated with these medications.

Anti-TNF- $\alpha$ agents may induce sudden vasculitis neuropathy at any time during treatment which must be distinguished from the neuropathy of the underlying disease. Appropriate therapy should be initiated immediately and continued long enough.

\section{Conflict of Interests}

All authors deny any conflict of interests related to this manuscript.

\section{References}

1. Kollias G, Douni E, Kassiotis G, Kontoyiannis D. The 
function of tumour necrosis factor and receptors in models of multi-organ inflammation, rheumatoid arthritis, multiple sclerosis and inflammatory bowel disease. Ann Rheum Dis. 1999;58(Suppl 1):I32-39.

2. Hamilton K, Clair EW. Tumour necrosis factor-alpha blockade: a new era for effective management of rheumatoid arthritis. Expert Opin Pharmacother. 2000;1(5):1041-1052.

3. Baker DE. Adalimumab: human recombinant immunoglobulin g1 anti-tumor necrosis factor monoclonal antibody. Rev Gastroenterol Disord. 2004;4(4):196-210.

4. Berthelot CN, George SJ, Hsu S. Distal lower extremity paresthesia and foot drop developing during adalimumab therapy. J Am Acad Dermatol. 2005;53(5 Suppl 1):S260-262.

5. Bensouda-Grimaldi L, Mulleman D, Valat JP, AutretLeca E. Adalimumab-associated multiple sclerosis. J Rheumatol. 2007;34(1):239-240; discussion 240.

6. Kieseier BC, Kiefer R, Gold R, Hemmer B, Willison HJ, Hartung HP. Advances in understanding and treatment of immune-mediated disorders of the peripheral nervous system. Muscle Nerve. 2004;30(2):131-156.

7. Hartung HP. Immune-mediated demyelination. Ann Neurol. 1993;33(6):563-567.

8. Redford EJ, Hall SM, Smith KJ. Vascular changes and demyelination induced by the intraneural injection of tu- mour necrosis factor. Brain. 1995;118 ( Pt 4)(869-878.

9. Hall SM, Redford EJ, Smith KJ. Tumour necrosis factor-alpha has few morphological effects within the dorsal columns of the spinal cord, in contrast to its effects in the peripheral nervous system. J Neuroimmunol. 2000;106(1-2):130-136.

10. Stubgen JP. Tumor necrosis factor-alpha antagonists and neuropathy. Muscle Nerve. 2008;37(3):281-292.

11. Chung JH, Van Stavern GP, Frohman LP, Turbin RE. Adalimumab-associated optic neuritis. J Neurol Sci. 2006;244(1-2):133-136.

12. Makol A, Grover M. Adalimumab induced mononeuritis multiplex in a patient with refractory rheumatoid arthritis: a case report. Cases J. 2008;1(1):287.

13. Richette P, Dieude P, Damiano J, Liote F, Orcel P, Bardin T. Sensory neuropathy revealing necrotizing vasculitis during infliximab therapy for rheumatoid arthritis. J Rheumatol. 2004;31(10):2079-2081.

14. Lozeron P, Denier C, Lacroix C, Adams D. Long-term course of demyelinating neuropathies occurring during tumor necrosis factor-alpha-blocker therapy. Arch Neurol. 2009;66(4):490-497.

15. Guillevin L, Mouthon L. Tumor necrosis factor-alpha blockade and the risk of vasculitis. J Rheumatol. 2004;31(10):1885-1887. 